\title{
Stochastic fields to analyze uncertainties in laminated structures with viscoelastic core
}

\author{
William P. Hernández, Daniel A. Castello ${ }^{1}$ and Thiago G. Ritto \\ ${ }^{a}$ Department of Mechanical Engineering. Universidade Federal do Rio de Janeiro. \\ Rio de Janeiro. RJ. Brasil \\ Received on March 11, 2018 / Accepted on Aug 10, 2018
}

\begin{abstract}
The process of polymeric materials production may lead to material heterogeneities. Therefore, the mechanical behavior of these materials may present unique characteristics from point to point. Nevertheless, this characteristic is rarely taken into account inasmuch as, in general, most model building strategies assumes material homogeneity when performing inverse analysis. Furthermore, the dimensions of the test specimens may not be appropriate to evaluate the levels of heterogeneity within the material. This work investigates the impact of viscoelastic material heterogeneities when these are used to build laminated structures commonly used as passive vibration components. The viscoelastic behavior is modeled by means of a fractional derivative constitutive equation. The heterogeneity of the media is modeled by means of stochastic fields. Two types of correlations are considered to model the covariance matrices. A numerical application is presented for a three-layer laminated structure with a viscoelastic core.
\end{abstract}

Keywords: Viscoelasticity, Stochastic Fields, Laminated Structures, Damping, Uncertainty Quantification.

\section{Introduction}

The use of viscoelastic materials (VEM) are quite common in different fields of Engineering such as aeronautical, civil, mechanical and off-shore. This may be attributed to some characteristics such as low mass, low stiffness and its high capacity to dissipate mechanical energy. Concerning energy dissipation, it is a quite desirable characteristic when designing structural components working under dynamic loads. Therefore, this type of material is commonly used in passive control strategies.

The conception and implementation of passive control strategies usually demand the use computational models (CM). The CM of complex systems are generally built from bottom-up approaches. Therefore, a computational

\footnotetext{
${ }^{1}$ E-mail Corresponding Author: castello@mecanica.ufrj.br
} 
model is firstly built for each structural subcomponent. A key aspect to be taken into consideration is the fact that the more complex the engineering structure the greater the levels of uncertainty when building the final CM.

The theory of probability is one way to treat modeling uncertainties. Based on this theory, the uncertainty of any model parameter $\theta$ may be described by means of a probability density function (pdf) $\pi(\theta)$. Further, correlation among model parameters $\left\{\theta_{1}, \ldots, \theta_{N}\right\}$ may be described by the information present in a joint pdf $\pi\left(\theta_{1}, \ldots, \theta_{N}\right)$. Concerning uncertainties in material modeling, assuming that $\left\{\theta_{1}, \ldots, \theta_{N}\right\}$ are constitutive model parameters eventually leads to a stochastic model to describe the material behavior.

Hernadéz et al. [1] analyzed the uncertainties of modal parameters of a three-layer laminated structure when a stochastic model is used to describe the viscoelastic behavior of the middle layer. The stochastic model considers a constitutive equation based on fractional derivative operators. In [ref Hernandez et al] it is assumed material homogeneity. Nevertheless, the process of polymeric materials production of a component $\mathcal{B}$ may naturally lead to material heterogeneities. Therefore, the mechanical behavior of these materials may present unique characteristics from point $\mathbf{x}$ to point $\mathbf{x}^{\prime}$ within the body $\mathcal{B}$. A comprehensive analysis concerning the uncertainties of constitutive viscoelastic models should take this heterogeneity into consideration.

The contribution of this work is to present an general approach for describing the uncertainties associated to viscoelastic material behavior. Spatial heterogeneities are naturally taken into account by modeling the constitutive model parameters $\left\{\theta_{1}, \ldots, \theta_{N}\right\}$ as random fields, i.e., $\theta_{j}: \mathcal{B} \mapsto \mathbb{R}$. The theory of Gaussian random fields is used to generate a set of realizations $\left\{\theta_{1}^{(r)}, \ldots, \theta_{N}^{(r)}\right\}$ for the constitutive model parameters. Two types of functions are used to describe the spatial correlation between $\theta_{j}(\mathbf{x})$ and $\theta_{j}\left(\mathbf{x}^{\prime}\right)$.

\section{Constitutive Model}

The main characteristics of viscoelastic behavior may be described by appropriate constitutive models. In particular, there are some models based on the use of fractional derivative operators [2] . The general form for 1-D constitutive equation for an isotropic VEM based on time domain fractional derivative operators can be expressed as follows [2]

$$
\sigma(x, t)+b_{1} \frac{d^{\beta_{1}}}{d t^{\beta_{1}}} \sigma(x, t)+b_{2} \frac{d^{\beta_{2}}}{d t^{\beta_{2}}} \sigma(x, t)+\ldots+b_{n} \frac{d^{\beta_{n}}}{d t^{\beta_{n}}} \sigma(x, t)=
$$




$$
a_{0} \varepsilon(x, t)+a_{1} \frac{d^{\alpha_{1}}}{d t^{\alpha_{1}}} \varepsilon(x, t)+a_{2} \frac{d^{\alpha_{2}}}{d t^{\alpha_{2}}} \varepsilon(x, t)+\ldots+a_{m} \frac{d^{\alpha_{m}}}{d t^{\alpha_{m}}} \varepsilon(x, t)
$$

where $\left\{a_{1}, \ldots a_{m}\right\}$ and $\left\{b_{1}, \ldots b_{n}\right\}$ are constitutive model parameters, $t$ is the time, $0<\beta_{r} \leq 1$ and $0<\alpha_{s} \leq 1$ are the fractional derivative orders for the stress $\sigma(x, t)$ and strain $\varepsilon(x, t)$ fields, respectively. If $\left\{\beta_{r}, \alpha_{s}\right\}$ are integer numbers, Eq.1 leads to a classical constitutive model for an isotropic VEM [3]. For the sake of conciseness, the argument $x$ associated to the spatial variable in Eq.1 will be omitted henceforth. As for the fractional derivative operator $d^{\gamma} / d t^{\gamma}$ used in Eq.1, it is defined as follows

$$
\frac{d^{\gamma}}{d t^{\gamma}}[v(t)] \equiv \frac{1}{\Gamma(1-\gamma)} \frac{d}{d t} \int_{0}^{t} \frac{v(t-\bar{\tau})}{\bar{\tau}^{\gamma}} d \bar{\tau}
$$

where $\Gamma(\cdot)$ is the Gamma function and $\gamma$ is the order of the fractional derivative operator, $0 \leq \gamma \leq 1$. In this work it is used a model with five parameters described by Eq.3

$$
\sigma(t)+\tau^{\beta} \frac{d^{\beta}}{d t^{\beta}} \sigma(t)=G_{0} \varepsilon(t)+G_{0} \tau^{\beta} \frac{d^{\beta}}{d t^{\beta}} \varepsilon(t)+\left(G_{\infty}-G_{0}\right) \tau^{\alpha} \frac{d^{\alpha}}{d t^{\alpha}} \varepsilon(t)
$$

where $\tau$ is a relaxation time; and $G_{0}$ and $G_{\infty}$ are the static and dynamic material modulus, respectively.

Rewiriting Eq.3 in the frequency domain leads to a relation between stresses $\tilde{\sigma}(j \omega)$ and strains $\tilde{\varepsilon}(j \omega)$ using the concept of the complex modulus $\tilde{G}(j \omega)[3]$ as follows :

$$
\tilde{\sigma}(j \omega)=G_{0}\left(1+(d-1) \frac{(j \omega \tau)^{\alpha}}{1+(j \omega \tau)^{\beta}}\right) \tilde{\varepsilon}(j \omega)=\tilde{G}(j \omega) \tilde{\varepsilon}(j \omega)
$$

where $d=G_{\infty} / G_{0}$ is the ratio between the dynamic and static modulus and $\tilde{G}(j \omega)$ denotes the complex modulus of the material which is a frequency dependent operator.

It should be emphasized that the constitutive equation described by 4 presents the relation between stress $\sigma$ and strain $\varepsilon$ for each material point $x$ within a viscoelastic body $\mathcal{B}$. In the great majority of applications the constitutive parameters $\left\{G_{0}, d, \tau, \alpha, \beta\right\}$ are assumed as constants throughout the body domain. In other words, the hypothesis of homogeneous media is usually assumed when modeling viscoelastic materials. However, in practice, the production processes of polymeric materials generally lead to heterogeneities. Therefore, investigations about the impact of these heterogeneities on the material behavior is an issue of concern for engineering 
designs. In this context, next we present an approach to model spatial variability of the parameters $\left\{G_{0}, d, \tau, \alpha, \beta\right\}$ that are used to characterize the constitutive equation 4 . For this purpose, the theory of random fields is used.

\section{Random Fields}

Random fields $\left\{\lambda(\mathbf{x}): \mathbf{x} \in \mathbb{D} \subset \mathbb{R}^{n}\right\}$ are families of random variables which vary in spatial domain $\mathbb{D}$. Random fields may present different characteristics which is amenable to modeling distinct media. One may describe its characteristics by its mean value $\mathbb{E}[\lambda(\mathbf{x})]$ as well as its covariance $\operatorname{Cov}\left[\lambda(\mathbf{x}), \lambda\left(\mathbf{x}^{\prime}\right)\right]$.

In the context of material modeling, the theory of random fields is amenable to be used in the description of heterogenous properties of a body $\mathcal{B}$. Material heterogeneities may stem from production processes, for example. In this work the material heterogeneity in $\mathcal{B}$ will be described by means of the theory of random fields. The characterization of the random fields will be given by its mean value $\mathbb{E}[\lambda(\mathbf{x})]$ as well as its covariance $\operatorname{Cov}\left[\lambda(\mathbf{x}), \lambda\left(\mathbf{x}^{\prime}\right)\right]$ $\forall \mathbf{x}, \mathbf{x}^{\prime} \in \mathcal{B}[5]$.

In the present work, the constitutive parameters $\left\{G_{0}, d, \tau, \alpha, \beta\right\}$ in Equation 3 are modeled as stationary random fields. If $\lambda(\mathbf{x})$ is a stationary random field then the following holds

$$
\begin{gathered}
\mathbb{E}[\lambda(\mathbf{x})]=\mu_{\lambda} ; \quad \forall \mathbf{x} \in \mathcal{B} \\
\operatorname{Cov}\left[\lambda(\mathbf{x}), \lambda\left(\mathbf{x}^{\prime}\right)\right]=\mathbb{C}\left(\mathbf{x}-\mathbf{x}^{\prime}\right) ; \quad \forall \mathbf{x}, \mathbf{x}^{\prime} \in \mathcal{B}
\end{gathered}
$$

for which Eq.5 means that the mean of the field is constant throughout the body $\mathcal{B}$ and Eq. 6 means the the covariance matrix of the field depends only on the relative position of the material points $\mathbf{x}$ and $\mathbf{x}^{\prime}[5]$.

In order to generate a set of realizations $\left\{\lambda^{(1)}(\mathbf{x}), \ldots, \lambda^{(m)}(\mathbf{x})\right\}$ of the random field $\{\lambda(\mathbf{x}): \mathbf{x} \in \mathcal{B}\}$, a discrete version the Karhunen-Loève in finite dimensions is used [5]. This approach proved amenable for the present applications.

As for the covariance matrix, the applications consider two possibilities; namely, Gaussian and exponential [5]. The Gaussian covariance matrix $C_{g}$ is defined as follows

$$
C_{g}\left(\mathbf{x}, \mathbf{x}^{\prime}\right)=\sigma^{2} \exp \left(-\frac{\left\|\mathbf{x}-\mathbf{x}^{\prime}\right\|_{2}^{2}}{L_{c}^{2}}\right), \quad L_{c}>0
$$




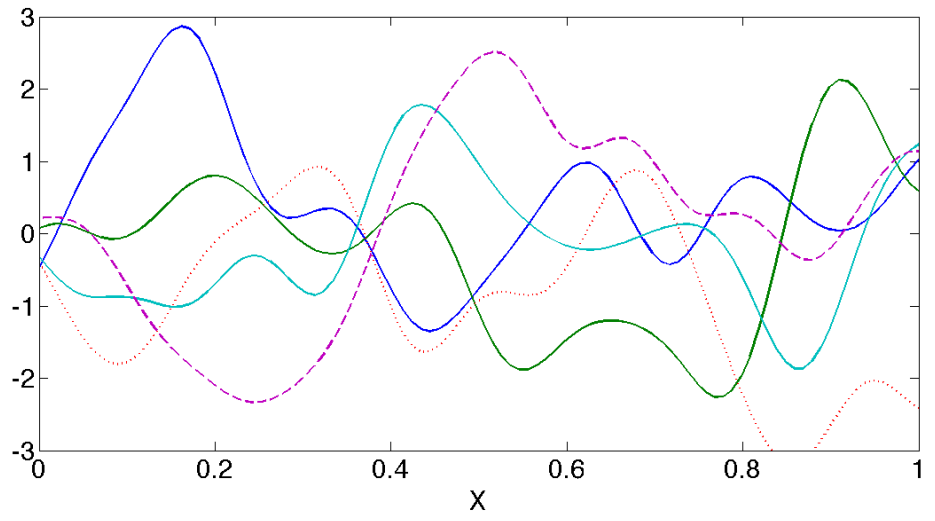

Figure 1: Realizations of a random field $\lambda(x)$ with: $\mathbb{E}[\lambda(x)]=0$ and Gaussian covariance matrix with $\sigma=1$ and correlation length $L_{c}=1 / 10$.

in which $L_{c}$ corresponds to a correlation length. As for the exponential covariance matrix $C_{\text {exp }}$, it is defined as follows

$$
C_{\text {exp }}\left(\mathbf{x}, \mathbf{x}^{\prime}\right)=\sigma^{2} \exp \left(-\frac{\left\|\mathbf{x}-\mathbf{x}^{\prime}\right\|_{2}}{L_{c}}\right), \quad L_{c}>0
$$

Figure 1 presents some realizations for a zero-mean random field with a Gaussian correlation with $\sigma=1$ and correlation length $L_{c}=0.1$. Figure 2 presents some realizations for a zero-mean random field with an exponential correlation with $\sigma=1$ and correlation length $L_{c}=0.1$. Finally, Figure 3 present the exact and the empirical correlation matrices computed with $10^{4}$ realizations.

\section{Applications}

Next an application is presented in which a laminated structure is the system under analysis

\subsection{System}

The physical system corresponds to a three-layer laminate structure. The outer layers are metallic and the core is made of a viscoelastic material. A sketch of the laminate structure is shown in Fig.4. The laminated system is the same as the one analyzed by Hernández et al. [1]. 


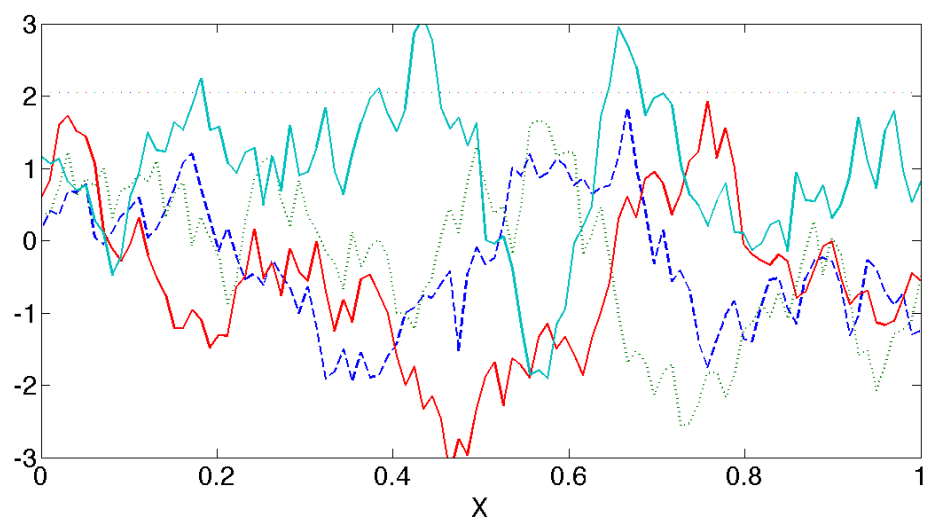

Figure 2: Realizations of a random field $\lambda(x)$ with: $\mathbb{E}[\lambda(x)]=0$ and exponential covariance matrix with $\sigma=1$ and correlation length $L_{c}=1 / 10$.
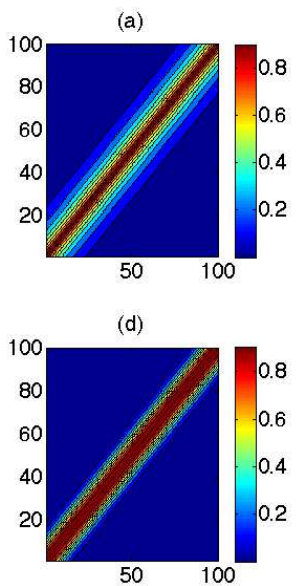

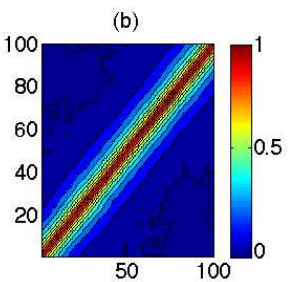

(e)

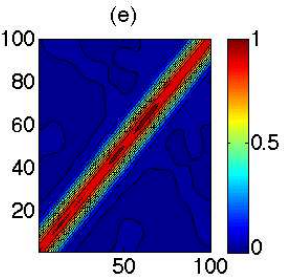

(c)
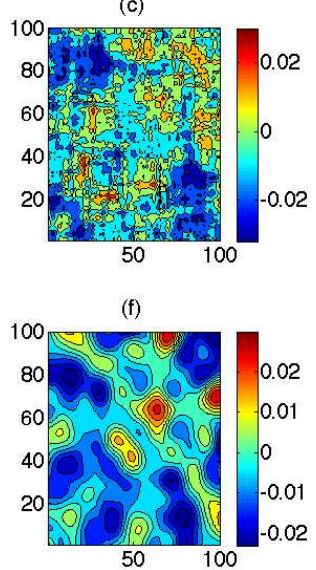

Figure 3: (a) Gaussian correlation matrix $C_{g}$ (exact). (b) Empirical Gaussian correlation matrix $C_{g}^{e m p}\left(10^{4}\right.$ realizations). (c) $C_{g}-C_{g}^{e m p}$. (d) Exponential correlation matrix $C_{\text {exp }}$ (exact). (e) Empirical exponential correlation matrix $C_{e x p}^{e m p}$ (10 $10^{4}$ realizations). (f) $C_{e x p}-C_{e x p}^{e m p}$. 


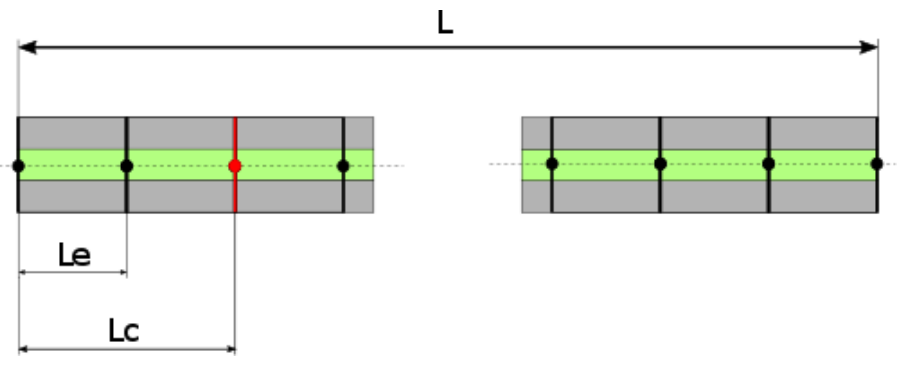

Figure 4: A sketch of a laminate structure of length $L . L_{e}$ : length of a finite element. $L_{c}$ : correlation length used for the covariance matrices of the random fields.

\subsection{Uncertainties}

The constitutive parameters $\left\{G_{0}, d, \tau, \alpha, \beta\right\}$ of the models used to describe the core of the laminates structure shown in Fig. 4 is considered. Figures 5 and 6 present the mean values and the standard deviation of the frequencies $\omega_{n}$ and modal damping factors $\zeta_{n}$ of the five first modes of the structure. It can be observed that expected values of the modal parameters do not vary as the correlation length changes. Nevertheless, it can be observed that the standard deviation of the modal parameters increase as the correlation length $L_{c}$ increases until a saturation value. It should be remarked that for this range of saturation the model provides basically uniform properties along the viscoelastic layer inasmuch as the correlation length is much greater than the beam length.

These preliminary results indicates that the expected value of modal parameters remain approximately constant for the lower modes when material heterogeneities are taken into account. There was no difference in the results when the Gaussian or exponential correlations were considered. As for the standard deviations, they presented an increase with the correlation length $L_{c}$ until a certain limit. All these analysis are valid for the lower modes. Further investigations are currently under work in order to extend these analysis.

\section{Concluding Remarks}

The present work presents an approach to take material heterogeneities into account when analyzing viscoelastic media. The first analysis indicate its suitability when treating laminated structural components that are com- 

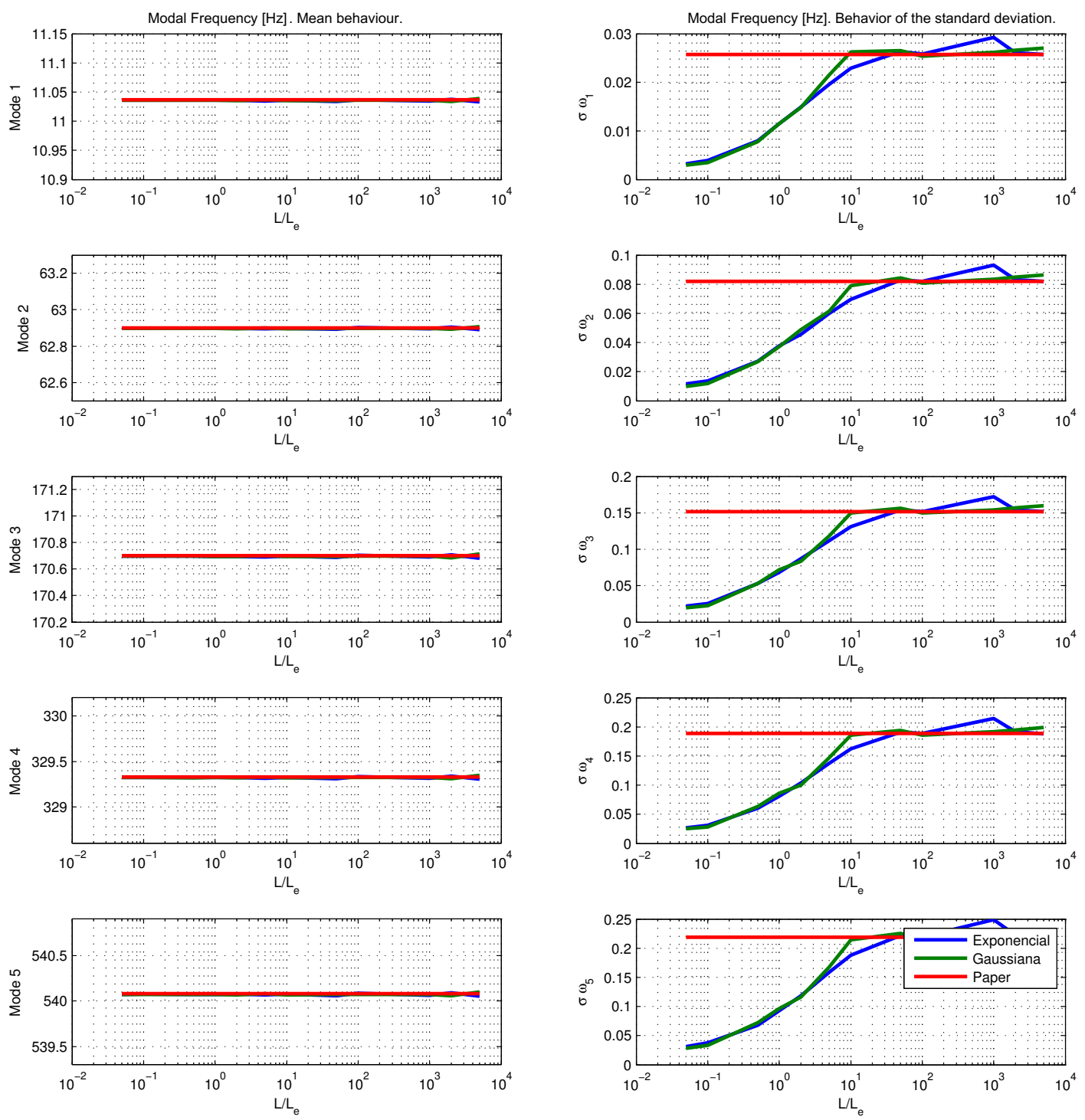

Figure 5: The mean and variance of the first five natural frequencies as a function of the ratio between the correlation length $L_{c}$ and the finite element length $L_{e}$. 

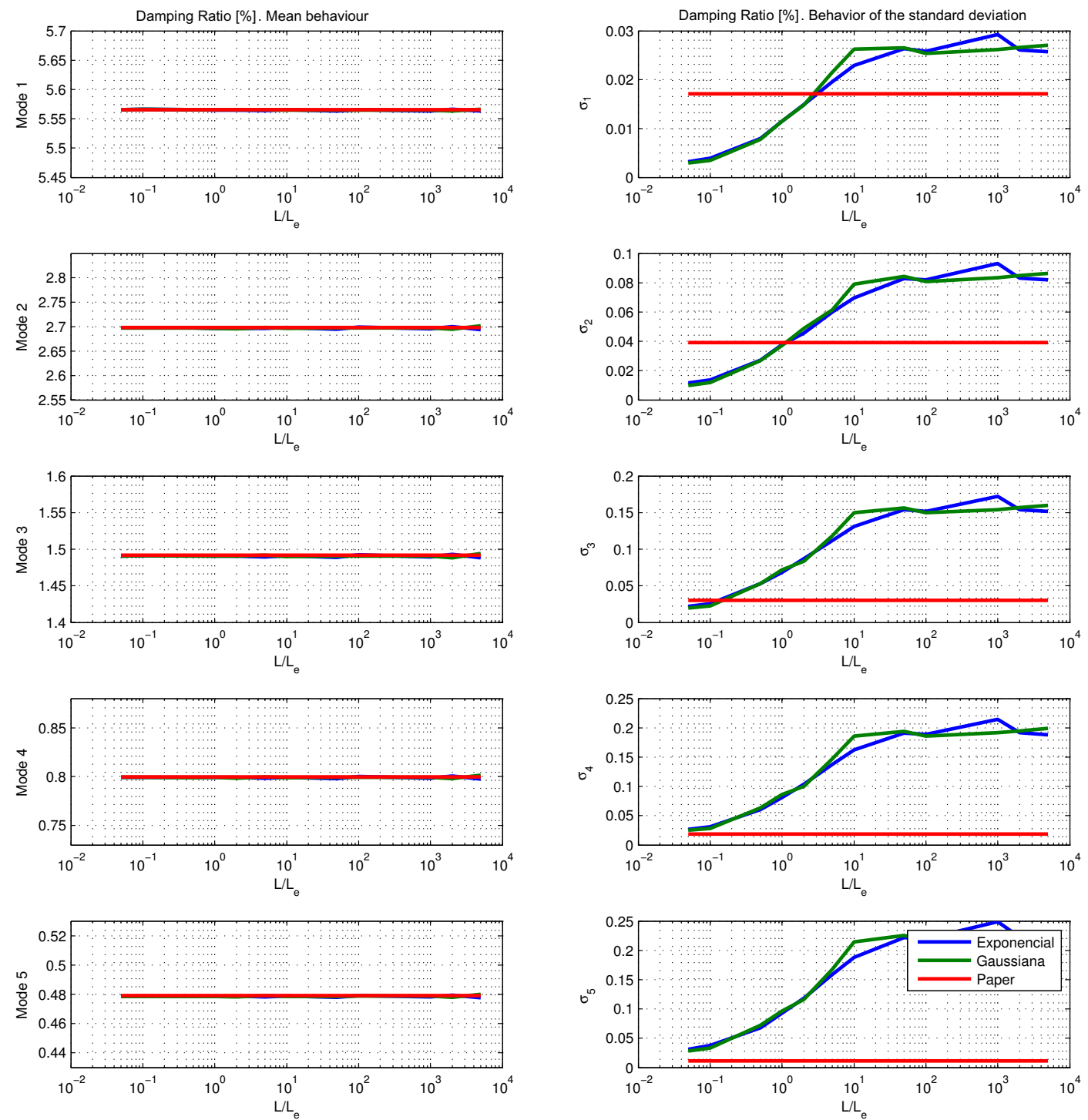

Figure 6: The mean and standard deviation of the first five damping factors as a function of the ratio between the correlation length $L_{c}$ and the finite element length $L_{e}$. 
monly used for passive vibration control.

Acknowledgments. The authors would like to express their gratitude to the National Council for Scientific and Technological Development (CNPq) for its financial support under grant number 483391/2013, to Coordenação de Aperfeiçoamento de Pessoal de Nível Superior (CAPES) for its financial support under grant number AUXPE n.1197/2014.

\section{References}

[1] Hernández WP, Castello DA and Ritto TG. Uncertainty propagation analysis in laminated structures with viscoelastic core, Computers \& Structures, 164(1), 2016, 23-37.

[2] Fractional calculus - A different approach to the analysis of viscoelastically damped structures, AIAA Journa, 21(5), 1983, 741-748.

[3] Wineman, A.S and Rajagopal, K.R., Mechanical Response of Polymers: An Introduction, Cambridge University Press, Cambridge, England ; New York, 2000.

[4] Pritz, T., Five-parameter fractional derivative model for polymeric damping materials, Journal of Sound and Vibration, n5(v265), 2003, 935-952.

[5] Lord GJ, Powell CE and Shardlow T. An Introduction to Computational Stochastic PDEs, 2014, Cambridge Texts in Applied Mathematics.

doi: $10.6062 /$ jcis.2018.09.03.0152

(c) Author(s) 2019. This work is distributed under

the Creative Commons Attribution 4.0 License.

cc) (i) 